Int. J. Dev. Biol. 55: 467-476

doi: $10.1387 / \mathrm{ijdb} .103224 \mathrm{sl}$

\title{
Current concepts of blood-brain barrier development
}

\author{
STEFAN LIEBNER ${ }^{*}, 1$, CATHRIN J. CZUPALLA ${ }^{1}$ and HARTWIG WOLBURG ${ }^{2}$ \\ ${ }^{1}$ Blood-Brain Barrier Signaling Group, Institute of Neurology, Edinger-Institute, Medical School, Goethe University \\ Frankfurt and ${ }^{2}$ Institute of Pathology, Medical School, Eberhard-Karls University Tübingen, Germany
}

\begin{abstract}
Homeostasis of the central nervous system (CNS) microenvironment is essential for its normal function and is maintained by the blood-brain barrier (BBB). The BBB proper is made up of endothelial cells (ECs) interconnected by tight junctions (TJs) that reveal a unique morphology and biochemical composition of the body's vasculature. In this article, we focus on developmental aspects of the BBB and describe morphological as well as molecular special features of the neurovascular unit (NVU) involved in barrier induction. Recently, we and others identified the Wnt/ $\beta$ catenin pathway as crucial for brain angiogenesis, TJ and BBB formation. Based on these findings we discuss other pathways and molecular interactions for BBB establishment and maintenance. At the morphological level, our concept favors a major role for polarized astrocytes (ACs) therein. Orthogonal arrays of particles (OAPs) that are the morphological correlate of the water channel protein aquaporin-4 (AQP4) are specifically formed in the membrane of the AC endfoot. The polarized AC endfoot and hence OAPs are dependent on agrin and dystroglycan, of which agrin is a developmentally regulated extracellular matrix (ECM) component. Understanding the mechanisms leading to BBB development will be key to the understanding of barrier maintenance that is crucial for, but frequently disturbed, in the diseased adult brain.
\end{abstract}

KEY WORDS: development, blood-brain barrier, aquaporin-4, Wnt pathway, endothelial cell

\section{Introduction}

The original finding of Paul Ehrlich that an infused dye did not stain the brain tissue, together with the complementary observation of his associate Edwin E. Goldmann that the very same dye if applied into the cerebrospinal fluid did exclusively stain the brain tissue, has lead to the concept of a biological barrier between blood and brain (for review see Liebner and Engelhardt, 2005). The term "blood-brain barrier" was coined by Lewandowsky, who observed that neurotoxic compounds led to neuronal cell death only if applied directly into the brain, but not after systemic injection into the vasculature (for review see Liebner and Engelhardt, 2005). However, the cellular basis of the blood-brain and the blood-cerebrospinal fluid barrier was unclear for decades. Today, we know that in most vertebrates the BBB is located within the endothelium (endothelial BBB; only in elasmobranchs, the BBB is located in ACs) and in the epithelial choroid plexus cells and the tanycytes of the circumventricular organs (CVOs; glial blood-cerebrospinal fluid barrier [BCSFB]) (Abbott et al., 2010). The structures essentially responsible for the restriction and control of the paracellular flux between epithelial and endothelial cells were identified as TJs. Originally, these intercellular contacts were studied exclusively by means of morphological methods such as tracing experiments using electron-dense tracers like lanthanum nitrate or horseradish peroxidase, and freeze-fracturing. From the middle of the 1980s on, the molecular organization of the TJs was unraveled step by step, but primarily in epithelial cells. Today, we overlook a multitude of molecules involved in the formation as well as the regulation of barrier properties, but we are far from understanding the molecular interplay, which represents the transcellular barrier. The regulation of the endothelial BBB TJs seemingly is more complex than that of epithelial cells. In culture, the latter but not ECs, develop TJs with all morphological, molecular and physiological features, which are known from their counterparts in vivo. Therefore, it is of upmost importance to understand in detail how endothelial barrier properties develop and which cellular and molecular prerequisites have to be available. It has become likely that BBB tightness is not just "switched on" at a specific time point during brain angiogenesis but

\footnotetext{
Abbreviations used in this paper: AC, astrocyte; BBB, blood-brain barrier; BCSFB, blood-cerebrospinal fluid barrier; BL, basal lamina; BRB, blood-retina barrier; CNS, central nervous system; CVOs, circumventricular organs; EC, endothelial cell; NPC, neural precursor cell; NVU, neuro-vascular unit; OAPs, orthogonal arrays of particles; PC, pericyte; PNVP, perineural vascular plexus; TJ, tight junction.
}

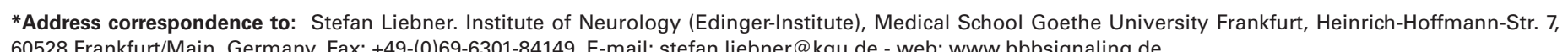
60528 Frankfurt/Main, Germany. Fax: +49-(0)69-6301-84149. E-mail: stefan.liebner@kgu.de - web: www.bbbsignaling.de 
rather that the tightening of the barrier occurs as a gradual process, which is independent from vascular proliferation and begins during embryogenesis when angiogenesis is not complete (for review see Liebner and Engelhardt, 2005). On the other hand, it should be stressed that an unequivocal correlation between tight junction structure and permeability has not been shown so far (Saunders et al., 2008). It is now clear that BBB endothelial TJs are elements responsive to the brain microenvironment in a complex manner that includes the surrounding basal lamina $(\mathrm{BL})$ as well as the "second line of defense" consisting of pericytes (PCs), ACs, and microglia. Together with interneurons, making occasional contact to the endothelium, this unique cellular composition is defined as the NVU (Fig. 1). Although hundreds and thousands of research papers have been published over the years describing the BBB, only little is known about the signal transduction from the $A C$ to the $E C$ or possibly from the $E C$ to the $A C$ to induce and maintain barrier properties. And this only concerns the mature situation and its maintenance. Kim and co-workers representatively demonstrated

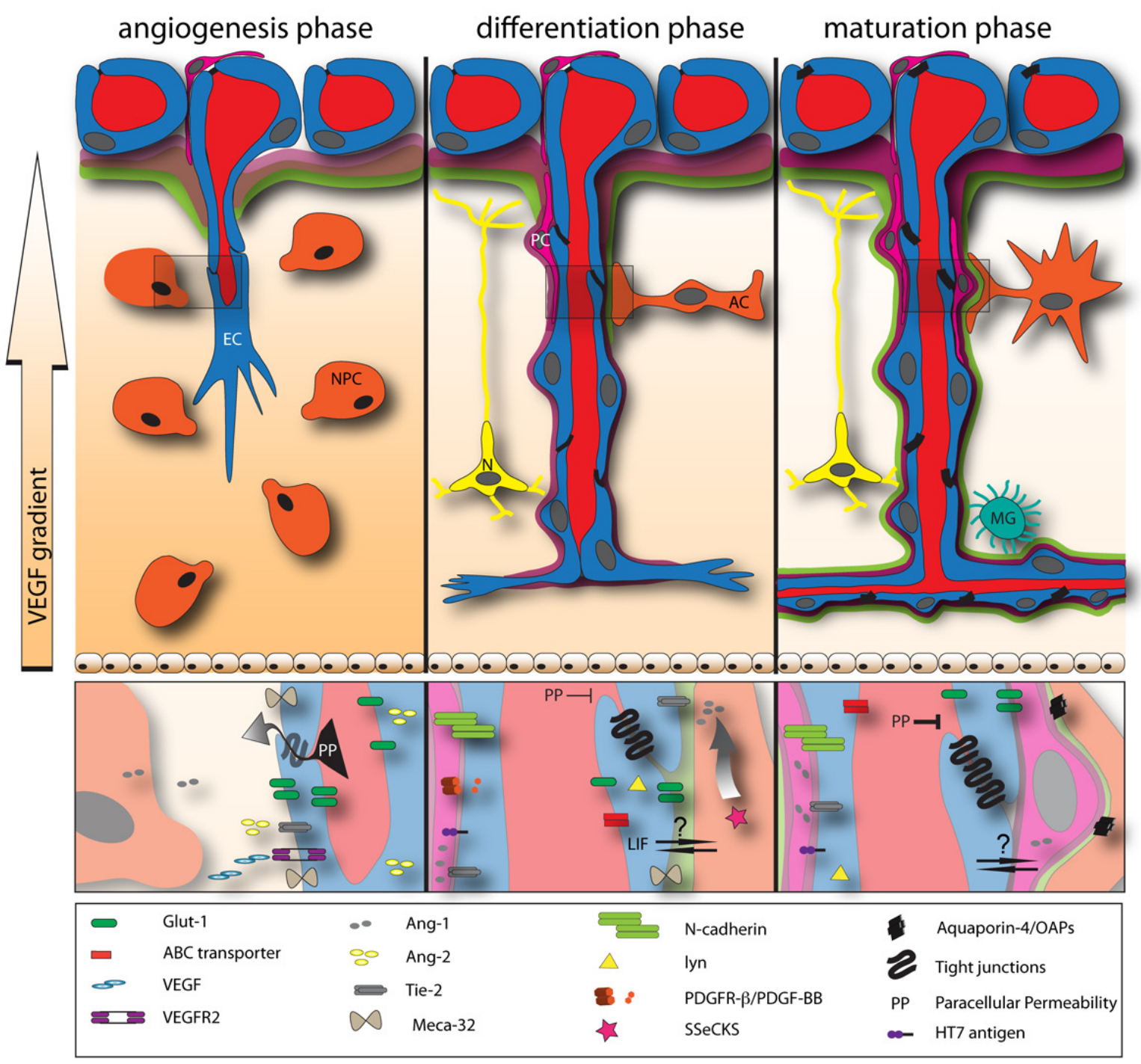

Fig. 1. Differentiation of the Blood-brain barrier (BBB). Angiogenesis phase: Vascular sprouts radially invade the embryonic neuroectoderm towards a concentration gradient of VEGF-A, which is produced by neuroectodermal cells located in the ventricular layer. VEGF-A binds to its endothelial receptor, the receptor tyrosine kinase fIk-1/KDRNEGFR2. The EC specific receptor tyrosine kinase Tie-2 and its ligand Ang-1 are involved in angiogenic sprouting early during embryogenesis. The cerebral ECs show Glut-1 evenly distributed and the MECA-32 antigen is highly expressed, contributing to poor barrier characteristics and high paracellular permeability (PP). Differentiation phase: The phenotype of cerebral ECs changes such that they downregulate expression of the MECA-32 antigen. Glut-1 is now enriched on the abluminal surface of the endothelium. De novo expression of ABC transporters and the nonreceptor tyrosine kinase lyn can be observed. The TJs become complex and thus tight for small polar molecules. Phenotypic changes of ECs are accompanied by their close contact with PCs and astroglial cells. Recruitment of PCs along the differentiating BBB vessels is ensured by several mechanisms. PDGF-BB produced by ECs binds to its receptor PDGFR- $\beta$ on PCs; N-cadherin enriched at sites of PC-EC contact; Ang-1 expressed by PCs binds to the endothelial receptor tyrosine kinase Tie-2. ECs produce LIF, inducing the maturation of ACs via the LIF-R 3 . Furthermore, increased oxygen level and EC-derived PDGF-BB lead to an upregulation of SSeCKS in ACs that in turn upregulates Ang-1. Maturation phase: Despite the fact that the cerebral ECs form the barrier proper, close contact with PCs, ACs and maybe neuronal cells is required for the maintenance of the BBB. The molecular mechanisms involved in this crosstalk required for BBB maintenance in the mature CNS remain unknown to date. 
the formation of the endothelial blood-retina barrier (BRB) TJs to be characterized by a close interplay of ECs with PCs and ACs (Kim et al., 2009). In this chapter, we will focus on the development of the BBB, but we will start with the description of the structure and function of the brain microvascular ECs in the mature system, in order to have a basis for looking at earlier stages.

\section{Phenotype of the mature blood-brain barrier}

The BBB faces the conflict to protect - on the one side - the microenvironment of the CNS from toxins and fluctuations in blood composition, and-on the other side - to deliver energy substrates and nutrients to the CNS. Nature has resolved this issue by combining barrier with transportmechanisms. The subcellular barrier structures are constituted by endothelial TJs, whereas delivery mechanisms are represented by a plethora of receptors and transporters, first of all the glucose transporter isoform 1 (Glut1). The cells responsible for establishing the barrier and for providing all receptors and transporters are the capillary ECs in case of the BBB, and the epithelial (glial) cells in case of the blood-cerebrospinal fluid barrier.

\section{The epithelial (glial) cells of the blood-cerebrospinal fluid barrier}

The epithelial (glial) cells include the ependymal cells of the brain ventricles, the choroid plexus epithelial cells, the tanycytes of the circumventricular organs, the pituicytes in the hypothalamichypophyseal system and the pigment epithelial cells in the retina. As non-neuronal, neuroectodermal cells they are clearly defined as a subtype of glial cells, responsible for homeostasis in the CNS and for the formation of the BCSFB and the outer BRB.

The choroid plexus epithelial cells, the tanycytes and the retinal pigment epithelial cells are underlined by a BL that distinguishes them from the "normal" cilia-bearing ependymal cells lining the major part of the ventricular surface. The apical surface of ependymal cells is, at least in mammals, characterized by marked regional variations throughout the ventricles. Ependymal cells consistently carry groups of kinocilia, intermingled with microvilli of variable frequency and size. On the contrary, in choroid plexus epithelial cells microvilli are frequent and extended in length, while single cilia or groups of cilia are rarely found. At their basal pole, choroid plexus epithelial cells form an extended basal labyrinth and are situated on a BL delimiting the inner stroma of connective and highly vascularized tissue. The ECs of the choroid plexus blood vessels are constitutively fenestrated in order to warrant high permeability. The fenestration is known to be induced by vascular endothelial growth factor (VEGF), and represents the physiological basis for the capability of the choroid plexus epithelial cells to produce the cerebrospinal fluid (CSF) from the blood. To prevent the diffusion of blood-borne substances into the ventricle, choroid plexus epithelial of OAPs (encircled area). cells are equipped with TJs. These TJs are the site of the BCSFB proper (for a recent review on choroid plexus, see Wolburg and Paulus, 2010).

\section{The endothelial cell}

The phenotype of mature BBB capillaries in the mammalian brain first of all can be characterized by the small height of ECs, the interendothelial TJs, the small number of caveolae at the luminal surface of the cell, and the high number of endothelial mitochondria (for review see Liebner and Engelhardt, 2005). In the capillary bed of the vasculature, there is only one BLbetween the EC and the AC or the PC. ECs of precapillary arterioles and postcapillary venules have an own BL, which is separated from the glial one. The space between both basal laminae is called the perivascular space and is continuous with the Virchow-Robin space at the surface of the brain.

TJs in general represent contact zones between cells or between parts of the same cell (as it is the case in BBB ECs), in which the intercellular cleft is occluded ("Zonula occludens"). In ultrathin sections, TJs are recognized as a chain of fusion ("kissing") points, which appear, in freeze-fracture replicas, as transversally sectioned strands forming more or less complicated networks (Fig. 2 A-C). The complexity of the meshwork inversely correlates with the permeability and thus with the stage of development (Wolburg and Lippoldt, 2002). Using the freeze-fracture method, the membrane halves can be observed in the transmission electron microscope from the interior of the cell giving the aspect of the external fracture face or E-face, or from outside the cell, giving the aspect of the internal or protoplasmic fracture face or P-face (Fig. 2 D-I). Concerning the BBB EC TJs, they were found to be the most complex ones in the whole vasculature of the body. But also the association of the particles with the P-face or E-face is a parameter that allows estimating the quality of the TJs. The BBB TJs are unique among all endothelial TJs given that their P-face association is as high as or even slightly higher than their E-face

\section{A angiogenesis phase}


E13.0
B
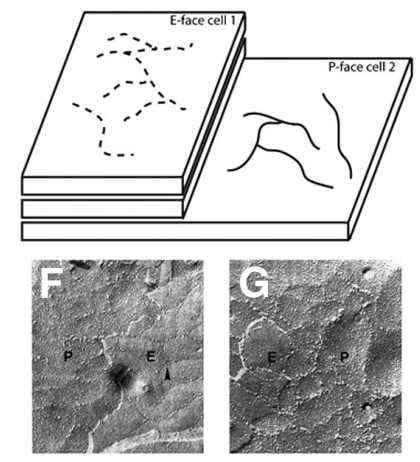

E15.0

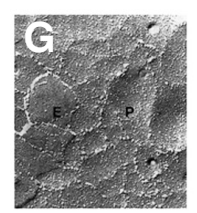

E18.0
C maturation phase
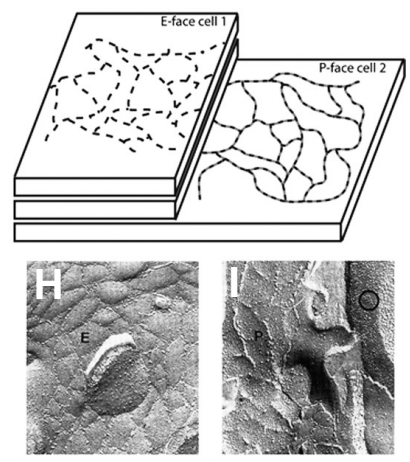

P1
Fig. 2. Development of tight junction morphology in brain endothelial cells. TJ morphology develops in parallel to brain angiogenesis, vessel differentiation and maturation. (A) During the angiogenesis phase (E13.0), only particle-free grooves on the E-face and ridges on the P-face can be observed in freeze fracture preparations. (B) In the differentiation phase (E15.0, E18.0), the network of E-and P-face structures becomes more complex and increasingly filled with particles. (C) The postnatal maturation phase (P1) is characterized by a highly complex network of strands and particle enriched E-face grooves (40\% particles) and P-face strands (60\% particles). The latter frequently show continuous ridges that may represent residual membrane structures. Note in (I) the astrocyte endfoot appose to the endothelium, revealing high density 
association. Interestingly, in cell culture, where the brain-specific microenvironment is absent, the freeze-fracture morphology of BBB ECs is similar to that of non-BBB ECs in vivo (Wolburg and Lippoldt, 2002). During development of the BBB in the rat, the Pface association of the EC TJs continuously increases, indicating that the association of strand particles with the inner membrane leaflet reflects the quality of the barrier and is under the control of intrinsic brain factors (Fig. 2).

The molecular biology of TJs has been summarized elsewhere (reviewed in Wolburg and Lippoldt, 2002). Most data were generated in epithelial cells, probably because the regulation of BBB endothelial TJs might be considerably more complex than that in epithelial cells. On the other side, most TJ molecules including the scaffolding proteins mediating between the TJ proper and the cytoskeleton were not described in ECs so far. Briefly, after the detection of TJ-associated proteins such as ZO-1, ZO-2, ZO-3 and cingulin, intramembrane proteins were found to be directly involved in the restriction of the interendothelial permeability. The first protein found was occludin, a protein with four transmembrane domains; however, occludin seemed not to be the most important permeability restricting TJ component, since the occludin-deficient mouse mutant is viable and has intact biological barriers. The discovery of the claudins, a new gene family of TJ-related proteins, which also have four transmembrane domains but no homology to occludin (for review see Wolburg and Lippoldt, 2002) was of fundamental importance for TJ research. In addition, members of the immunoglobulin superfamily, such as the junctional adhesion molecules (JAMs) and the endothelial selective adhesion molecule (ESAM), are components of TJs, which probably fulfill regulatory functions of the barrier. The exciting field of the regulation of TJs by adherens junctions was outlined several times (Dejana etal., 2009).

\section{The pericyte}

PCs are integral constituents of each capillary with different frequencies in different vascular beds. At the electron microscopical level, PCs can easily be recognized by being completely surrounded by a BL. Immunohistochemically, they can be labeled by antibodies against smooth muscle actin, desmin, high molecular weight melanoma antigen (HMW-MMA, called NG2 in the mouse), plateletderived growth factor $\beta$-receptor (PDGFR- $\beta$ ), aminopeptidase $\mathrm{N}$ or the regulator of G-protein signalling-5 (RGS5). In the CNS, and in particular in the retina, PCs are most abundant. There is a plethora of studies on the role of PCs during embryonic angiogenesis (see below) and on metabolic interdependencies between ECs and $\mathrm{PCs}$ concerning the regulation of blood flow in brain and retina or the maturation of the blood-brain or blood-retina barrier (for review see Krueger and Bechmann, 2010). It should be noted that recent work suggests that PCs play a crucial role in BBB establishment as discusses in the later chapters.

\section{The astrocyte}

ACs constitute the most numerous cell class in CNS. In order to nominate only some of the functions of ACs, they are involved in physiological and biochemical tasks like compartmentalization of the neural parenchyma, maintenance of the ionic homeostasis of the extracellular space, $\mathrm{pH}$ regulation, neurotransmitter uptake and processing, providing energy rich substrates to the neurons and the mediation of signals from neurons to the vasculature (for a recent comprehensive review, see Sofroniew and Vinters,
2010). The direct interface between the neuroglial and the vascular compartment is established by the perivascular glial endfeet in the NVU. Therefore, it is not surprising that in recent literature these endfeet have been stressed as key checkpoints of brain metabolism (Wolburg et al., 2009).

In our context, it is of particular importance that ACs are believed to play a decisive role in the maintenance of the barrier properties of the brain microcapillary ECs and in controling the cerebral blood flow (Abbott et al., 2010).

One of the most impressive morphological features of mammalian and avian ACs is the polar heterogeneity of astrocytic membrane domains. Where the astroglial endfeet contact the superficial or perivascular BL(glia limitans superficialis et perivascularis), the glial membrane contains numerous square arrays or intramembranous OAPs (Wolburg et al., 2011). Where the glial cell membrane loses the contact with the BL and turns into deeper areas of the neuropil, the density of OAPs is dramatically reduced. This kind of astroglial polarity seems to correlate with the BBB maturation during development. Now, it has become clear that OAPs contain the water channel protein AQP4. Consequently, it has been searched for the significance of AQP4 for the integrity of the BBB in AQP4 knock out mice. However, the data are not unequivocal and at present investigations provide contradictory data (Wolburg et al., 2011).

Aquaporins mediate water movements between the intracellular, interstitial, vascular and ventricular compartments, which are under the strict control of osmotic and hydrostatic pressure gradients (for review see Amiry-Moghaddam and Ottersen, 2003). Interestingly, the inward rectifier potassium channel Kir4.1, the $\mathrm{K}^{+}$conductivity, and the dystrophin-dystroglycan complex (DDC) are colocated with AQP4. Dystrophin is a scaffolding protein linking the membrane and the cytoskeleton. In dystrophin-deficient mice, Nico and co-workers observed an increase of the vascular permeability, a loss of some tight junctional components, and a reduction in the expression of AQP4 (Nico et al., 2003).

\section{Maintenance of the blood-brain barrier}

As we have seen before, the direct interface between the neuroglial compartment and the vascular compartment is established by the perivascular glial endfeet, forming the glial limiting border. The temporal correlation between astroglial differentiation and BBB maturation during embryonic development suggests a functional role of ACs for establishment and/or maintenance of the BBB.

In this context the role of agrin for BBB maintenance is of particular interest. Agrin is an extracellular heparan sulfate proteoglycan that was originally characterized as the essential molecule for clustering acetylcholine receptors at the motor endplate (Bezakova and Ruegg, 2003), but has also been described as being important within the CNS, particularly for the integrity of the BBB (Berzin et al., 2000). Several agrin splicing variants have been characterized: AOB0 was reported to be specifically present in the endothelial cell $\mathrm{BL}$ of CNS capillaries, whereas neurons express isoforms with inserts at the B splicing site (Kroger and Schroder, 2002). Agrin $A 0 B 0$, when present in AC primary cultures, had no effect on the expression level of AQP4 nor on the water transport capacity in hypotonic challenge experiments (Noell et al., 2009). However, agrin $\mathrm{AOB} 0$ was able to induce increased insertion of AQP4 into the membrane of cultured ACs.

The mechanism of how agrin influences BBB maintenance is not 
understood so far. Agrin may indirectly regulate AQP4 distribution in the glial membrane (Wolburg etal., 2009). It binds to $\alpha$-dystroglycan, which is a member of the receptor family "deleted in colorectal cancer" (DCC) (Gee et al., 1994), but not directly to AQP4. Due to the close connection between AQP4 and $\alpha$-dystroglycan, loss of agrin went along with a redistribution of $A Q P 4$ and finally with a loss of OAPs and OAP-related polarity. Supporting this observation, we recently could show that in the newborn agrin-knockout mouse the astrocytic endfeet were not devoid of AQP4, but of OAPs (Wolburg et al., 2011) suggesting that AQP4 can also occur in a non-array


Fig. 3. Molecular organization of the neurovascular unit (NVU) and the role of Wnt signaling in brain endothelial cells (ECs). In the brain and spinal cord mainly Wnt7a and Wnt7b growth factors act on an uncharacterized Fzd-LRP receptor complex to elicit $\beta$-catenin target gene transcription. The positively regulated target genes identified so far are the TJ protein claudin-3, Glut-1 and ABCB1/MDR1. Conversely, the expression of Meca-32/ Plvap becomes suppressed by $\beta$-catenin transcription via an unknown mechanism. See the grey supported area and arrows for the "canonical" Wnt pathway. In the mature BBB, AC endfeet are almost entirely covered by OAPs, formed by AQP4 that can only be visualized in their structure by freeze fracture electron microscopy (right). AQP4 is linked to $\alpha$-/ $\beta$-dystroglycan that in turn binds to agrin and Kir4. 1. Intracellular, the complex is bound to the actin cytoskeleton via dystrophin. 
form. Thus, since, without any exception, all BBB formations in the adult and sound mammalian brain are associated with a high OAP-related polarity of perivascular astrocytic endfeet, this seems to be important for the integrity of the BBB (Fig. 3). In turn, the high OAP-related polarity of ACs seems to be, at least in part, dependent on the presence of agrin.

Another role of agrin in the maintenance of the BBB concerns the stability of the molecular composition of BBB endothelial TJs. In the human glioblastoma, four types of microvessels were described, which were characterized by different amounts of agrin, tenascin, occludin and two claudins. If agrin was completely absent, tenascin was highly expressed, and the TJ molecules were lost. However, we do not know so far whether agrin directly influences the composition of TJs, or whether the disturbance of TJs is an indirect consequence of the reduced AC polarity driven by loss of agrin (as explained above).

\section{Development of the brain vasculature}

The development of the head vasculature is initiated when migration of mesodermal hemangioblasts gives rise to the formation of so-called blood islands, which in turn differentiate by fusion into the primary vascular plexus. This process is designated as vasculogenesis, the de novo formation of blood vessels. Subsequently, the primitive capillary network expands by proliferation and sprouting, an event described as angiogenesis, which in contrast to vasculogenesis entails the growth of new blood vessels from pre-existing ones (for review see Liebner and Engelhardt, 2005).

Notably, the central nervous system acquires its vasculature solely by angiogenesis, a process composed of EC proliferation, sprouting and migration. At about embryonic day 9 in rodents the vessels of the perineural vascular plexus (PNVP) cover the entire brain and start to grow radially along a preformed lattice of radial glial and neural precursor cells (NPCs) into the neuroectodermal tissue, forming an undifferentiated network of capillaries (Virgintino et al., 1998) (Fig. 1). These NPCs give rise to differentiated neurons and $\mathrm{ACs}$, which will dynamically communicate to the brain endothelium to form a regular and fully functional NVU (Anthony et al., 2004).

A decade ago the vascularization of the CNS was thought to be a passive event, driven by the requisition of the developing brain tissue for nutrients and oxygen. More recently it has been shown that cell autonomous signals seem to trigger both, brain blood vessel and neuronal development (Vasudevan et al., 2008). Additionally, it is known that the brain vascularization proceeds according to a tightly regulated spatial and temporal program, which reaches its climax at early postnatal stages.

In the 1980s W. Risau addressed the question what makes ECs grow into the neuroectoderm. The first paracrine factors that were thought to be the main inducers of endothelial sprouting into the neuroectoderm were the acidic and basic fibroblast growth factors (aFGF, bFGF). Even though they were known to be strong inducers of angiogenesis in vivo in general, it turned out that their expression pattern and those of their corresponding receptors did not fit to the spatio-temporal occurrence of angiogenic processes within the brain (for review see Liebner and Engelhardt, 2005).

The most prominent vascular endothelial growth inducer is VEGF, a paracrine factor, which in particular activates endothelial proliferation, survival and sprouting via binding to its receptors VEGF receptor 1 (VEGFR1, flt-1) and VEGF receptor 2 (VEGFR2, flk-1,
KDR) (Carmeliet, 2005). VEGF plays a major role in the stimulation of angiogenesis within the development of the brain by guiding ECs along a concentration gradient from the pial surface to the ependymal cell layer (Fig. 1). Vessel formation and growth has also been shown to be dependent on various other growth factor-receptor entities such as angiopoietins (Ang-1, Ang-2) and their common receptor Tie2. The Ang-Tie system is responsible for the recruitment of perivascular cells and is also involved in vascular sprouting and remodeling besides adhesion to the ECM (Augustin et al., 2009). Both VEGF and Ang-1 were discovered to be expressed in motor neurons localized in close vicinity of the neuroectoderm invading vessels, suggesting the involvement in angiogenic sprouting. By inhibiting the induction of these motor neurons in mouse embryos using cyclopamine, an inhibitor of sonic hedgehog (Shh) signaling, vessel sprouting was dramatically impaired indicating that the neural tube angiogenesis is dependent on Shh signaling, which seems to be mediated by Ang-1 positive motor neurons (Nagase et al., 2005). Also in another context the inhibition of Shh resulted in reduced physiological retinal angiogenesis and inhibition of pathological vascularization (Surace et al., 2006).

Likewise the platelet derived growth factor B (PDGF-BB) seems to play a definite role in $\mathrm{PC}$ recruitment as deficient mice completely lack PC coverage of the brain vessels. Beside PDGF signaling also direct contact between PCs and ECs during brain angiogenesis, mediated by the adherens junction receptor $\mathrm{N}$-cadherin (Fig. 1 and 3 ), seems to be crucial for proper endothelial growth and stability. As aforementioned, the PC coverage varies among different vessel types and is most abundant in the vessels of the CNS. For example the $P C$ to $E C$ ratio in the retina is $1: 1$, followed by a $1: 3$ ratio for brain vessels and a proportion of 1:100 in heart muscle vessels (Zlokovic, 2008). Although PC distribution and abundance suggested a specific role of this cell type in the BBB until recently, direct in vivo evidence has been lacking. Armulik et al. (2010), as well as Daneman et al. (2010) could show that impairment of PC coverage via the genetic ablation of PDGF-BB, leads to BBB leakiness, that however was not mediated by the disruption of endothelial cellcell junctions. This impressively underlines the importance of the interplay between different cell types for the proper development and maintenance of brain blood vessels.

Additionally it has been shown that the heterodimeric $\alpha \mathrm{v} \beta 8$ integrin and its ligand, the latent transforming growth factor $\beta$ (TGF $\beta$ ), are critical for embryonic neurovascular development, as an ablation of one of the integrin subunits leads to morphological abnormalities in brain blood vessels structure and neurovascular malformations (Zhu et al., 2002). $\alpha \mathrm{V} \beta 3$-integrin is expressed by ACs and not by brain ECs, suggesting that paracrine communication of ACs and ECs could collaborate to gain and sustain brain vessel integrity. Indeed, it turned out that the described neurovascular disorders including intracerebral hemorrhages and deformed blood vessels are due to the missing integrin-mediated activation of TGF- $\beta$, which in turn inhibits downstream activation of TGF- $\beta$-responsive genes e.g. plasminogen activator inhibitor-1 (PAl-1) and thrombospondin-1 (TSP-1) which are known to promote vessel differentiation and stabilization (Cambier et al., 2005).

With respect to vascular sprouting DIl4/Notch1 signaling was identified to be a key mechanism, coordinating the tip and stalk cell pattern during angiogenic sprouting. Phng et al. could show that the Notch-regulated ankyrin repeat protein (Nrarp) acts as a molecular link between Notch- and Lef1-dependent Wnt signaling 
in ECs (reviewed in Phng and Gerhardt, 2009). Wnt proteins are secreted glycoproteins which bind to the N-terminal cystein-rich domain (CRD) of seven-pass-transmembrane Frizzled (Fzd) receptors. $\beta$-Catenin is the main component of the canonical Wnt signaling pathway (Wnt/ $\beta$-catenin pathway), as a transcription factor mediating the regulation of cellular responses such as cell cycle, apoptosis, cell differentiation and communication (for review see Liebner and Plate, 2010) (Fig. 3). However, in contrast to the hematopoietic system, little is known about the role of Wnt signaling in ECs and during developmental angiogenesis in particular. But since a few years growing evidence points to a role of the Wnt pathway in angiogenesis and vascular homeostasis, given that several findings in vitro and in vivo showed that various functional vascular defects are due to the mutation of Wnt and Fzd genes (Zerlin et al., 2008). Especially, it became clear that the canonical pathway is crucial for proper murine neurovascular development (Fig. 3). In particular, the simultaneous ablation of the growth factors Wnt7a/7b leads to embryonic lethal neurovascular phenotypes, as it causes CNS-specific hemorrhages and disorganization of the neural tissue. Activation of the canonical Wnt signaling pathway is CNS specific, as it was not found in peripheral tissues including the heart, liver, and lung. Complementary, the Wnt/ $\beta$-catenin pathway is necessary and sufficient to induce BBB-type endothelial TJs in vitro and in vivo on a molecular and structural level (for review see Liebner and Plate, 2010).

\section{Differentiation of the blood-brain barrier and phenotypic changes}

During early development, the adhesion between ECs and PCs might be the result of the release of chemotactic factors by ECs to induce the migration of PCs towards the EC wall and subsequent maturation of the vessels by an increased production of extracellular matrix components elicited by the action of activated TGF- $\beta$ and other proteins (Lai and Kuo, 2005). Amongst these, PDGF-BB, a high affinity ligand for the receptor tyrosine kinase PDGF-R $\beta$ present on $P C s$ is produced by $E C$ s during development. PDGF-BB has been shown to be involved in vascularization of the brain, since the deletion of the PDGF-BB gene led to PC loss, endothelial hyperplasia, abnormal vascular morphogenesis, and lethal microaneurysm formation during late embryogenesis. More recently, it has been shown that deletion of the C-terminal PDGF retention motif by gene targeting led to a malformation of the PC investment. Since this retention motif binds heparansulfate proteoglycans, and the alteration of heparansulfate synthesis by defective $\mathrm{N}$-sulfation resulted in a detachment of PCs, it was suggested that heparansulfate is important for PDGF signaling during vascular development (reviewed in Gaengel et al., 2009).

Whether agrin is also among the heparansulfate proteoglycans modifying PDGF signaling, is currently not known.

As mentioned above, extensive $P C$ recruitment is one of the hallmarks of the functional BBB, but BBB differentiation comprises the interaction with additional cell types, namely ACs. ACs cover almost the entire surface of microcapillaries with their endfeet structures in the mature NVU. The formation of these endfeet is the consequence of the interaction with $\mathrm{ECs}$ and at the same time, $A C s$ via the endfoot structure foster EC differentiation and BBB maturation (Wolburg et al., 2009). Despite their widely accepted importance for the formation and maintenance of the BBB, little is known about the molecular dynamics of the intimate interaction between ECs and ACs. However, Lee and co-workers identified the potential tumor suppressor src-suppressed C-kinase substrate (SSeCKS, spoken "essex", human ortholog is gravin), which was upregulated in ACs by endothelial-derived PDGF-BB and downregulated astrocytic VEGF expression (Lee et al., 2003). Furthermore, SSeCKS markedly upregulated Ang-1, a known vascular maturation factor, leading to the strengthening of interendothelial junctions (Fig. 1). On the other hand, the differentiation of ACs from common neuroectodermal precursors is supported by the angiogenic endothelium that releases leukemia inhibitory factor (LIF), which in turn fosters AC differentiation. Mechanistically, LIF activates STAT3 in concert with retinoic acid (RA) and BMP2, which leads to the expression of glial fibrillary acidic protein (GFAP) (Fukushima et al., 2009). Additionally, the Notch pathway via Notch1-ICD and its downstream target Hes 5 could induce AC differentiation even more efficiently (Rodriguez-Rivera et al., 2009). As mentioned previously in the present review, the Notch pathway plays a fundamental role in angiogenic sprouting and endothelial differentiation. Notch1, that is expressed by stalk cells and activated by DII4 on tip cells, leads to the suppression of the angiogenic phenotype and hence to vessel stabilization (for review see Phng and Gerhardt, 2009). It is tempting to speculate that direct contact between NPCs and ECs may lead to Notch pathway activation in NPCs and subsequently to differentiation into ACs. Whether this hypothesis is true has to be tested in the future.

While the cellular components of the NVU find together, barrier properties of ECs develop in parallel. ECs sequentially lose vesicles and fenestrations, which might be the result of high VEGF-A levels at early embryonic stages. Furthermore, the early TJs, which are not more than particle-free grooves on the E-face and ridges on the P-face, consecutively acquire the molecular composition and morphology of the adult, characterized by claudin- 3 and claudin- 5 expression and a complex network of P-face associated particles (Wolburg et al., 2009) (Fig. 2).

The gain of barrier characteristics in ECs encompasses the loss of characteristic of leaky vessels. This is particularly intriguing for the plasmalemma vesicle associated protein (Plvap or Meca-32) (Hallmann et al., 1995). Although Meca-32 is also absent from some peripheral blood vessels in the adult, for example in those of skeletal muscle, its down regulation in the brain vasculature nicely correlates with claudin-3 expression and barrier maturation (Liebner et al., 2008). However, the mechanism by which Meca-32 is down regulated remains elusive.

Differentiation of the barrier phenotype comprises the upregulation of specific transporter systems mentioned in previous sections of this article. Most prominently, Glut-1 becomes upregulated and predominantly distributed at the ablumenal membrane in the differentiating BBB endothelium (Dobrogowska and Vorbrodt, 1999). However, as Simpson et al. (2001) pointed out, the method of how the distribution of Glut-1 was measured played a decisive role on the determination of the luminal to abluminal ratio (Simpson et al., 2001). Furthermore, other transporters, such as transferrin transporter as well as various amino acid transporters become specifically high expressed at the BBB. A separate class of BBBspecific transport systems is the family of multidrug resistance (ATP-binding cassette, ABC) transporters. These transporters, of which Mdr1/ABCB1b and ABCG2 are most prominently expressed, are considered as a second line of defense, responsible for the 
clearance of noxious compounds (Abbott et al., 2010). Interestingly, members of the P450 oxygenases, and in particular the isoform Cyp1b1, have been proposed to be highly expressed in ECs of the BBB (Dauchy et al., 2009). Although the regulation and function of Cyp1b1 at the BBB is unclear by know, it might also be important for preventing noxious xenobiotics from entering the brain via the metabolization of these substances.

\section{Molecular mechanisms of blood-brain barrier induction and differentiation}

By now the cellular and molecular structure of the BBB has been extensively described and many functional aspects of the BBB in relation to physiological, pathological and pharmacological characteristics have been elucidated. However, the knowledge of the cellular and molecular factors and pathways, which determine the entire spectrum of BBB-specific differentiations of the endothelium are still only superficially understood.

When brain angiogenesis starts, ECs inevitably get in contact with neuroectodermal cells like neuroblasts, and various glial precursors.

Recently, it has been shown that already vessels in the mouse perineural vascular plexus at embryonic day 9.5 receive signals of the Wnt pathway that participate in BBB induction (for review see Liebner and Plate, 2010). The analysis of the BAT-gal reporter mouse for $\beta$-catenin driven Wnt signaling, revealed that ECs of the PNVP, strongly express the LacZ reporter gene. Hence, induction of the BBB phenotype, likely by Wnt/ $\beta$-catenin signaling, parallels brain angiogenesis in the first sprouting vessels when the cell types of the brain are not yet differentiated (Fig. 1). Indeed, Wnt7a/b as the identified physiological Wnt growth factors, were shown to be required for CNS angiogenesis as well as for induction of barrier properties by regulating Glut1 expression (reviewed in Liebner and Plate, 2010). Whether Wnt growth factors are expressed by postnatal ACs or other differentiated, neuroectodermal cell types and if these factors exhibit barrier inducing/maintaining properties on ECs is matter of ongoing investigations.

In addition to the canonical Wnt pathway, the orphan G proteincoupled receptor (GPCR) 124/tumor endothelial marker 5 was recently identified as a major regulator of developmental CNS angiogenesis and endothelial barrier induction evidenced by upregulation of Glut-1. Beside the striking phenotype similarities to the Wnt7a/b knock out, a link or epistatic effect of GPCR124 with $\beta$-catenin has not been reported so far (Kuhnert et al., 2010).

This is further supported by previous publications, showing that NPCs provide BBB-inducing signals to ECs that however, are active during an early phase of barrier differentiation (Weidenfeller et al., 2007). During late embryonic and early postnatal development when neurons, and glial cell types start to differentiate, the quality of BBB inductive cues may change, suggested by the finding that conditioned medium from postnatal ACs have a delayed but longer lasting effect on BBB characteristics in primary brain ECs (Weidenfeller et al., 2007, and own unpublished results). Indeed, it could be demonstrated in vivo, that ACs are capable of inducing some BBB-characteristics in vessels of the anterior eye chamber, although the molecular identity of the astrocytic factors remains elusive (for review see Liebner and Plate, 2010). It is well accepted by now that the brain environment is sufficient to induce endothelial barrier properties. That ECs do not show a predetermination to the BBB-phenotype was elegantly shown by chick-quail xenografts in which vessels of the coelimic cavity of the embryonic chick acquired BBB characteristics when growing into the developing transplanted quail brain (reviewed in Liebner and Engelhardt, 2005).

Beside the neuroectodermal cells also PCs have been discussed to participate in the induction of barrier characteristics in ECs.

Dente et al. (Dente et al., 2001) and Nakagawa et al. (Nakagawa et al., 2007) have reported on PCs evoking an improvement of barrier properties in co-cultured microvessel ECs. In addition, PCs were shown to protect barrier properties under conditions of oxygen deprivation (Al Ahmad et al., 2009). On the other hand, Zozulya and collegues could recently demonstrate that PC conditioned medium or co-culture of PCs with porcine brain ECs increase the expression of the matrix metallo-protease 9 (MMP-9) in ECs, a marker for angiogenic vessel (Zozulya et al., 2008). Consequently, the transendothelial electrical resistance (TEER) of ECs was reduced by the PC co-culture, indicating reduced barrier properties in these cells. By now it is difficult to draw a clear picture of the role of PCs in BBB induction and maintenance. In particular, just as for postnatal ACs also for PCs the molecular mechanism behind these barrier-modulating properties remains elusive.

Apart from the Wnt pathway, which seems to play a major role in brain angiogenesis and BBB induction, other signaling circuits have been brought to light that modulate barrier properties.

Some evidence also points to a role of the ECM for normal brain vascular development and maintenance. In particular, laminin $\alpha 4$ seems to be crucial for the transmigration of $\mathrm{T}$ lymphocytes over the endothelium into the inflamed brain (Wu et al., 2009). Laminin $\alpha 4$ deficient mice ubiquitously upregulate laminin $\alpha 5$ along brain vessels, resulting in marked T lymphocyte infiltration into the brain parenchyma. Laminin $\alpha 5$ directly inhibits integrin $\alpha 6 \beta 1-$ mediated migration of $\mathrm{T}$ lymphocytes through laminin $\alpha 4$. These data point to the importance of the ECM in mediating endothelial permeability to transmigration of inflammatory cells and hence to BBB maintenance.

The activation of the dimeric growth factor PDGF-CC by the tissue plasminogen activator (tPA), which is essential for the thrombolytic treatment of ischemic stroke, caused an opening of the BBB by tPA (Su et al., 2008). PDGF-CC rather than plasminogen was identified as a substrate for tPA (Su et al., 2008) and intraventricular administration of tPA or PDGF-CC resulted in an increase of the BBB permeability, which could be counteracted by anti-PDGF-CC antibodies. Similarly, antagonizing the astroglialocated PDGF receptor $\alpha$ after ischemic stroke had comparable effects (Su et al., 2008). Thus, the activation of PDGF signaling by tPA seems to be involved in the deterioration of BBB integrity with all the known clinical complications of stroke.

\section{Outlook}

Since its first definition more than 100 years ago by Paul Ehrlich and Edwin Goldmann, the BBB has become a tremendously important functional structure of the vascular tree, which experiences increasing attention from various scientific disciplines. In particular, the development of BBB-passing pharmaceutical compounds for the treatment of neurological diseases is of upmost importance. As Western societies grow continuously older, the probability and incidence of age-related neurological diseases such as Alzheimer's disease $(A D)$, stroke and brain tumors there is an increasing demand for the treatment of these plagues. Academic scientists and pharmaceutical companies follow different strategies to address this issue. 
Beside the possibility to highjack BBB-specific transporters, also the modulation of paracellular barrier properties is one reasonable option. To follow the latter strategy, the profound understanding of the molecular inductive and maintaining mechanisms is crucial. In the last couple of years we made one major step forward in this process, by identifying the canonical Wnt pathway as a brain-specific angiogenic pathway that is key for the early induction of the BBB phenotype in ECs (for review see Liebner and Plate, 2010). Still, many questions remain unanswered as it is not clear by now of which nature the BBB-maintaining signals are, and which cell types are providing them. Furthermore, the Wnt pathway may participate directly or indirectly in the induction of additional barrier related genes than claudin-3 and Glut-1, which have been identified so far. In particular, active Wnt signaling in brain ECs, may influence the communication between ECs, ACs and PCs in the NVU, and may therefore participate in the formation of this unique and highly polarized cell-cell interactions.

As we have mentioned above, the heparansulfate proteoglycan agrin may play a decisive role in establishing the polarity of perivascular ACs that in turn may induce and maintain the BBB. The complexity of the regulatory processes at the BBB seems to be as advanced as the synaptic and even cognitive processes. Presumably, an increase of the BBB permeability is not necessarily an indication of pathological processes (stroke, inflammation, tumor, neurodegenerative diseases), but may rather be a brain-specific capability to access hematogenous factors in the circulation that modulate neural characteristics including turnover of adult neural stem cells. Investigating such processes would create a "vascular neurobiology". However, at present such subtle regulatory events are not detectable by any non-invasive imaging technique used in the clinical routine. Nevertheless, understanding all the endothelial, glial, pericyte and extracellular components of the BBB that may operate together at certain periods during development or during pathological conditions, will be the greatest challenge in the future of this growing, exciting, interdisciplinary field of research.

\section{Acknowledgements}

This study has been supported by the DFG SFB/TR23 grantB7 ("Vascular Differentiation and Remodeling", to SL), the Excellence Cluster CardioPulmonary System (ECCPS, to SL), the Onkogenic Signaling Frankfurt (OSF, LOEWE initiative Hessen, to SL), the EU FP7 consortium JUSTBRAIN (to $S L$ ) and by a grant of the Deutsche Krebshilfe (109219, to HW).

\section{References}

ABBOTT, N.J., PATABENDIGE, A.A.K., DOLMAN, D.E.M., YUSOF, S.R. and BEGLEY, D.J. (2010). Structure and function of the blood-brain barrier. Neurobiol. Disease 37: 13-25.

ALAHMAD, A., GASSMANN, M. and OGUNSHOLA, O.O. (2009). Maintaining bloodbrain barrier integrity: pericytes perform better than astrocytes during prolonged oxygen deprivation. J Cell Physiol 218: 612-622.

AMIRY-MOGHADDAM, M. and OTTERSEN, O.P. (2003). The molecular basis of water transport in the brain. Nat Rev Neurosci 4: 991-1001.

ANTHONY, T.E., KLEIN, C., FISHELL, G. and HEINTZ, N. (2004). Radial glia serve as neuronal progenitors in all regions of the central nervous system. Neuron 41:881-90.

ARMULIK, A., GENOVÉ, G., MÄE, M., NISANCIOGLU, M.H., WALLGARD, E., NIAUDET, C., HE, L., NORLIN, J., LINDBLOM, P., STRITTMATTER, K. et al. (2010). Pericytes regulate the blood-brain barrier. Nature 468: 557-561.

AUGUSTIN, H.G., KOH, G.Y., THURSTON, G. and ALITALO, K. (2009). Control of vascular morphogenesis and homeostasis through the angiopoietin-Tie system. Nat Rev Mol Cell Biol 10: 165-177.
BERZIN, T.M., ZIPSER, B.D., RAFII, M.S., KUO-LEBLANC, V., YANCOPOULOS, G.D., GLASS, D.J., FALLON, J.R. and STOPA, E.G. (2000). Agrin and microvascular damage in Alzheimer's disease. Neurobiol Aging 21: 349-355.

BEZAKOVA, G. and RUEGG, M.A. (2003). New insights into the roles of agrin. Nat Rev Mol Cell Biol 4: 295-308.

CAMBIER, S., GLINE, S., MU, D., COLLINS, R., ARAYA, J., DOLGANOV, G., EINHEBER, S., BOUDREAU, N. and NISHIMURA, S.L. (2005). Integrin alpha(v)beta8mediated activation of transforming growth factor-beta by perivascular astrocytes: an angiogenic control switch. Am J Pathol 166: 1883-1894.

CARMELIET, P. (2005). VEGF as a key mediator of angiogenesis in cancer. Oncology 69 Suppl 3: 4-10.

DANEMAN, R., ZHOU, L., KEBEDE, A.A. and BARRES, B.A. (2010). Pericytes are required for blood-brain barrier integrity during embryogenesis. Nature 468:562-566.

DAUCHY, S., MILLER, F., COURAUD, P.O., WEAVER, R.J., WEKSLER, B., ROMERO, I.A., SCHERRMANN, J.M., DE WAZIERS, I. and DECLEVES, X. (2009). Expression and transcriptional regulation of $A B C$ transporters and cytochromes P450 in hCMEC/D3 human cerebral microvascular endothelial cells. Biochem Pharmacol 77: 897-909.

DEJANA, E., TOURNIER-LASSERVE, E. and WEINSTEIN, B.M. (2009). The control of vascular integrity by endothelial cell junctions: molecular basis and pathological implications. Dev Cell 16: 209-221.

DENTE, C.J., STEFFES, C.P., SPEYER, C. and TYBURSKI, J.G. (2001). Pericytes augment the capillary barrier in in vitro cocultures. J Surg Res 97: 85-91.

DOBROGOWSKA, D.H. and VORBRODT, A.W. (1999). Quantitative immunocytochemical study of blood-brain barrier glucose transporter (GLUT-1) in four regions of mouse brain. J Histochem Cytochem 47: 1021-1030.

FUKUSHIMA, M., SETOGUCHI, T., KOMIYA, S., TANIHARA, H. and TAGA, T. (2009). Retinal astrocyte differentiation mediated by leukemia inhibitory factor in cooperation with bone morphogenetic protein 2. Int J Dev Neurosci 27: 685-690.

GAENGEL, K., GENOVÉ, G., ARMULIK, A. and BETSHOLTZ, C. (2009). Endothelialmural cell signaling in vascular development and angiogenesis. Arterioscl. Thrombo. Vasc. Biol. 29: 630-638.

GEE, S.H., MONTANARO, F., LINDENBAUM, M.H. and CARBONETTO, S. (1994). Dystroglycan-alpha, a dystrophin-associated glycoprotein, is a functional agrin receptor. Cell 77: 675-686.

HALLMANN, R., MAYER, D.N., BERG, E.L., BROERMANN, R. and BUTCHER, E.C. (1995). Novel mouse endothelial cell surface marker is suppressed during differentiation of the blood brain barrier. Dev Dyn 202: 325-332.

KIM, J.H., YU, Y.S., KIM, D.H. and KIM, K.W. (2009). Recruitment of pericytes and astrocytes is closely related to the formation of tight junction in developing retinal vessels. J Neurosci Res 87: 653-659.

KRÖGER, S. and SCHRÖDER, J.E. (2002). Agrin in the developing CNS: new roles for a synapse organizer. News Physiol Sci 17: 207-212.

KRUEGER, M. and BECHMANN, I. (2010). CNS pericytes: concepts, misconceptions, and a way out. Glia 58: 1-10.

KUHNERT, F., MANCUSO, M.R., SHAMLOO, A., WANG, H.-T., CHOKSI, V., FLOREK, M., SU, H., FRUTTIGER, M., YOUNG, W.L., HEILSHORN, S.C. et al. (2010). Essential regulation of CNS angiogenesis by the orphan $\mathrm{G}$ protein-coupled receptor GPR124. Science 330: 985-989.

LAI, C.H. and KUO, K.H. (2005). The critical component to establish in vitro BBB model: Pericyte. Brain Res Brain Res Rev 50: 258-265.

LEE, S.W., KIM, W.J., CHOI, Y.K., SONG, H.S., SON, M.J., GELMAN, I.H., KIM, Y.J. and KIM, K.W. (2003). SSeCKS regulates angiogenesis and tight junction formation in blood-brain barrier. Nat Med 9: 900-906.

LIEBNER, S., CORADA, M., BANGSOW, T., BABBAGE, J., TADDEI, A., CZUPALLA, C.J., REIS, M., FELICI, A., WOLBURG, H., FRUTTIGER, M. et al. (2008). Wnt/ beta-Catenin signaling controls development of the blood-brain barrier. $J$ Cell Biol 183: 409-417.

LIEBNER, S. and ENGELHARDT, B. (2005). Development of the blood-brain barrier. In The Blood-Brain Barrier and its Microenvironment: Basic Physiology to Neurological Diseases, vol. 1 (ed. PRAT, D. E. D. V. A. A.). Taylor and Francis Group, Boca Raton, FL, USA, Boca Raton, pp.1-26.

LIEBNER, S. and PLATE, K.H. (2010). Differentiation of the brain vasculature: the answer came blowing by the Wnt. J Angiogenes Res 2: 1.

NAGASE, T., NAGASE, M., YOSHIMURA, K., FUJITA, T. and KOSHIMA, I. (2005). 
Angiogenesis within the developing mouse neural tube is dependent on sonic hedgehog signaling: possible roles of motor neurons. Genes Cells 10: 595-604.

NAKAGAWA, S., DELI, M.A., NAKAO, S., HONDA, M., HAYASHI, K., NAKAOKE, R., KATAOKA, Y. and NIWA, M. (2007). Pericytes from brain microvessels strengthen the barrier integrity in primary cultures of rat brain endothelial cells. Cell Mol Neurobiol 27: 687-694.

NICO, B., FRIGERI, A., NICCHIA, G.P., CORSI, P., RIBATTI, D., QUONDAMATTEO, F., HERKEN, R., GIROLAMO, F., MARZULLO, A., SVELTO, M. et al. (2003). Severe alterations of endothelial and glial cells in the blood-brain barrier of dystrophic mdx mice. Glia 42: 235-251.

NOELL, S., FALLIER-BECKER, P., DEUTSCH, U., MACK, A.F. and WOLBURG, H. (2009). Agrin defines polarized distribution of orthogonal arrays of particles in astrocytes. Cell Tissue Res 337: 185-195.

PHNG, L.-K. and GERHARDT, H. (2009). Angiogenesis: a team effort coordinated by notch. Dev Cell 16: 196-208.

RODRIGUEZ-RIVERA, N.S., MOLINA-HERNANDEZ, A., SANCHEZ-CRUZ, E., ESCALANTE-ALCALDE, D. and VELASCO, I. (2009). Activated Notch1 is a stronger astrocytic stimulus than leukemia inhibitory factor for rat neural stem cells. Int J Dev Biol 53: 947-953.

SAUNDERS, N.R., EK, C.J., HABGOOD, M.D. and DZIEGIELEWSKA, K.M. (2008). Barriers in the brain: a renaissance? Trends Neurosci 31: 279-286.

SIMPSON, I.A., VANNUCCI, S.J., DEJOSEPH, M.R. and HAWKINS, R.A. (2001). Glucose transporter asymmetries in the bovine blood-brain barrier. J Biol Chem 276: 12725-12729.

SOFRONIEW, M.V. and VINTERS, H.V. (2010). Astrocytes: biology and pathology. Acta Neuropathol 119: 7-35.

SURACE, E.M., BALAGGAN, K.S., TESSITORE, A., MUSSOLINO, C., COTUGNO, G., BONETTI, C., VITALE, A., ALI, R.R. and AURICCHIO, A. (2006). Inhibition of ocular neovascularization by hedgehog blockade. Mol Ther 13: 573-579.

VASUDEVAN, A., LONG, J.E., CRANDALL, J.E., RUBENSTEIN, J.L. and BHIDE, P.G. (2008). Compartment-specific transcription factors orchestrate angiogenesis gradients in the embryonic brain. Nat Neurosci 11: 429-439.

Virgintino, D., MaIORANO, E., ERREDE, M., Vimercati, A., GReCo, P., SELVAGGI, L., RONCALI, L. and BERTOSSI, M. (1998). Astroglia-microvessel relationship in the developing human telencephalon. Int J Dev Biol 42: 1165-1168.

WEIDENFELLER, C., SVENDSEN, C.N. and SHUSTA, E.V. (2007). Differentiating embryonic neural progenitor cells induce blood-brain barrier properties. $J$ Neurochem 101: 555-565.

WOLBURG, H. and LIPPOLDT, A. (2002). Tight junctions of the blood-brain barrier: development, composition and regulation. Vascul Pharmacol 38: 323-337.

WOLBURG, H., NOELL, S., WOLBURG-BUCHHOLZ, K., MACK, A. and FALLIERBECKER, P. (2009). Agrin, aquaporin-4, and astrocyte polarity as an important feature of the blood-brain barrier. Neuroscientist 15: 180-193.

WOLBURG, H. and PAULUS, W. (2010). Choroid plexus: biology and pathology. Acta Neuropathol 119: 75-88.

WOLBURG, H., WOLBURG-BUCHHOLZ, K., FALLIER-BECKER, P., NOELL, S. and MACK, A. (2011). Structure and functions of aquaporin-4-based orthogonal arrays of particles. Int Rev Cell \& Mol Biol 287: 1-41.

WU, C., IVARS, F., ANDERSON, P., HALLMANN, R., VESTWEBER, D., NILSSON, P., ROBENEK, H., TRYGGVASON, K., SONG, J., KORPOS, E. et al. (2009). Endothelial basement membrane laminin alpha5 selectively inhibits $\mathrm{T}$ lymphocyte extravasation into the brain. Nat Med. 15: 519-527.

ZERLIN, M., JULIUS, M.A. and KITAJEWSKI, J. (2008). Wnt/Frizzled signaling in angiogenesis. Angiogenesis 11: 63-69.

ZHU, J., MOTEJLEK, K., WANG, D., ZANG, K., SCHMIDT, A. and REICHARDT, L.F. (2002). beta8 integrins are required for vascular morphogenesis in mouse embryos. Development 129: 2891-2903.

ZLOKOVIC, B.V. (2008). The blood-brain barrier in health and chronic neurodegenerative disorders. Neuron $57:$ 178-201.

ZOZULYA, A., WEIDENFELLER, C. and GALLA, H.-J. (2008). Pericyte-endothelial cell interaction increases MMP-9 secretion at the blood-brain barrier in vitro. Brain Res 1189: 1-11. 


\section{Further Related Reading, published previously in the Int. J. Dev. Biol.}

The seminal work of Werner Risau in the study of the development of the vascular system Domenico Ribatti

Int. J. Dev. Biol. (2010) 54: 567-572

Embryonic development of the proepicardium and coronary vessels Anna Ratajska, Elzbieta Czarnowska and Bogdan Ciszek Int. J. Dev. Biol. (2008) 52: 229-236

Vasculogenesis and angiogenesis in the mouse embryo studied using quail/mouse chimeras Michel Pudliszewski and Luc Pardanaud

Int. J. Dev. Biol. (2005) 49: 355-361

Vascular development: from precursor cells to branched arterial and venous networks Anne Eichmann, Li Yuan, Delphine Moyon, Ferdinand leNoble, Luc Pardanaud and Christiane Bréant Int. J. Dev. Biol. (2005) 49: 259-267

Parallels in invasion and angiogenesis provide pivotal points for therapeutic intervention Suzanne A. Eccles

Int. J. Dev. Biol. (2004) 48: 583-598

5 yr ISI Impact Factor $(2009)=3.253$

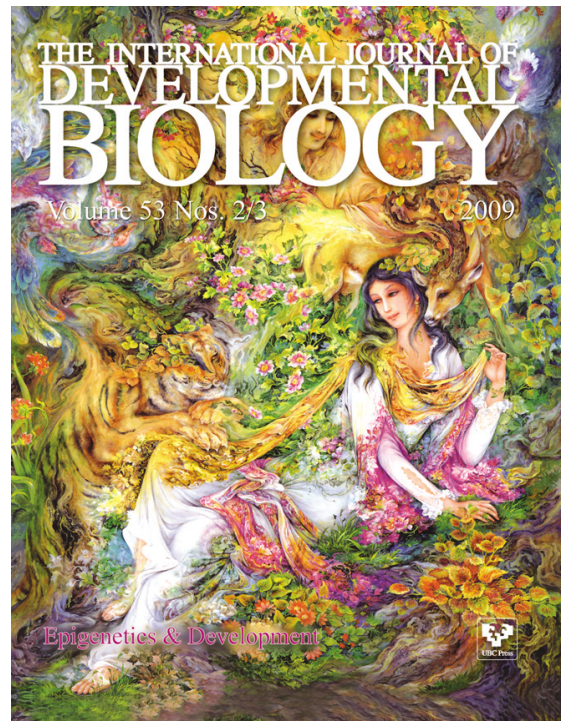

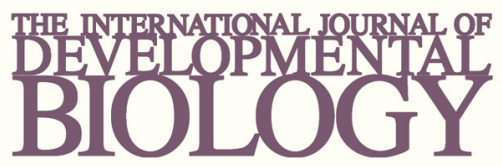

Volume 54 Nos. 6/7
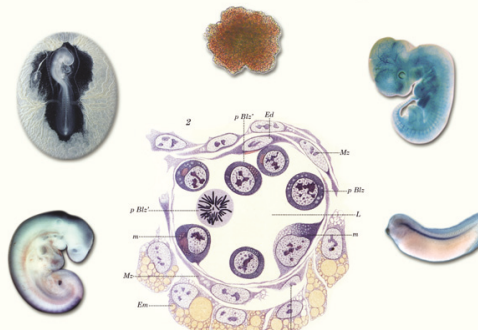

\section{年}


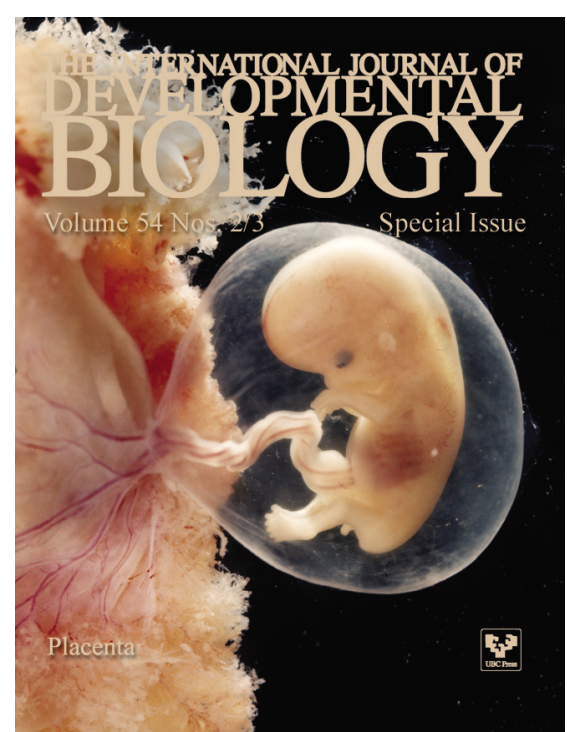

